\title{
Linking Sleep Slow Oscillations with consciousness theories: new vistas on Slow Wave Sleep unconsciousness
}

\author{
A. GEMIGNANI 1,2,3, D. MENICUCCl2,4, M. LAURINO2,3, A. PIARULLI5,6, \\ F. MASTORCl ${ }^{2}$, L. SEBASTIANI ${ }^{4}$, P. ALLEGRINI ${ }^{2,3,7}$ \\ ' Department of Surgical, Medical, Molecular \& Critical Area Pathology, University of Pisa, Pisa, Italy; \\ 2 Institute of Clinical Physiology, Research National Council, Pisa, Italy; ${ }^{3}$ Extreme Centre, Institute of \\ Life Sciences, Scuola Superiore Sant'Anna, Pisa, Italy; ${ }^{4}$ Department of Translational Research on New \\ Technologies in Medicine and Surgery, University of Pisa, Pisa, Italy; ${ }^{5}$ Perceptual Robotics Laboratory, \\ Scuola Superiore Sant'Anna, Pisa, Italy; ${ }^{\circ}$ Coma Science Group, GIGA-Research B34, University and \\ University Hospital of Liège; 7 Center for Nonlinear Science, University of North Texas, Denton TX U.S.A.
}

\section{A B S T R A C T}

We review current models of consciousness in the context of wakefulness and sleep. We show that recent results on Slow Wave Sleep, including our own works, naturally fit within consciousness models. In particular, Sleep Slow Oscillations, namely low-frequency $(<1 \mathrm{~Hz}$ ) oscillations, contain electrophysiological properties (up and down states) able to elicit and quench neural integration during Slow Wave Sleep. The physiological unconsciousness related to the Sleep Slow Oscillation derives from the interplay between spontaneous or evoked wake-like activities (up states) and half-a-second's electrical silences (down states). Sleep Slow Oscillation induces unconsciousness via the formation of parallel and segregated neural activities.

Key words

Slow Wave Sleep • Sleep Slow Oscillation •Unconsciousness $\bullet$ Models of consciousness $\bullet$ Brain criticality

This work is dedicated to the memory of Prof. Mario Guazzelli

\section{Introduction: NREM Sleep as a preferential phenomenon to study consciousness}

Although it is impossible to provide a definition of consciousness according to standard scientific reduction, neuroscientists have proposed empirical ways to establish levels of consciousness, from presence to absence. At present, consciousness is analytically studied through the various outputs correlating with it. These are called Neural Correlates of Consciousness (NCCs) (Tononi \& Koch, 2008). Deep, or dreamless, sleep is the only physiological state where consciousness fades, providing a window for defining plausible NCCs. Among these correlates, the ones that make more sense for defining consciousness are those that are constrained, by means of the so-called "backwards causation", by the global state of the brain (Gell-Mann, 2001; Feinberg, 2012).

Sleep electrophysiology includes several rhythms and waves that are peculiar of NREM sleep, the most important being the spindle waves, the delta waves and the Sleep Slow Oscillations (SSOs, including K Complexes), with a background of theta activity, that however can also be present during wakefulness, e.g. during meditative states (Cahn \& Polich, 
2006). The appearance of these peculiar rhythms is also accompanied with a modification of global "coherence", measured by connectivity indexes (Ferri et al., 2008). The vision that consciousness is an emerging property is corroborated by the complex structure of the "connectome". In other words consciousness may require the complex-network structure, from small world to scale-free properties, all being unraveled by an increasing corpus of scientific literature (see review of Sporns, 2014).

In this work, we shall focus on Slow Wave Sleep (SWS), namely the deepest stage of NREM sleep characterized by the occurrence of high amplitude oscillations, namely delta waves, and SSOs. The latter show distinctive negative peaks that are hallmarks, as we shall see, of electrical stillness in large neural assemblies (Amzica \& Steriade, 1998).

Finally, transitions towards and out of sleep-related unconsciousness may also be of help in understanding which rhythm and connectivity modifications can account for transient behaviors such as falling asleep and awakening. Herein, we will show some hints of these modifications by reporting our results on sensory processing during sleep, specifically on how it integrates neural activity in a wake-like fashion, before some sleep protection mechanism takes place. Our findings can also explain why people are fully aware of stimuli administered while they are asleep and unconscious, in case the sensoryprocessing arousal is strong enough.

The aim of the work is to provide new vistas on whether and how SSO fits within current theories of consciousness. To this aim the following Sections 2 and 3 respectively provide the background about theories of consciousness and SSO physiology. Section 4 outlines how sleep-related unconsciousness, in our opinion, obeys current theories of consciousness on the basis of recent experimental findings on SSOs.

\section{Theories of consciousness}

In this section we briefly review the current theories of consciousness, and how these may connect to a vision of brain complexity.

A prominent role is played by the notion of Baars' Global Workspace (GW) (1988, 1993). This is a psychological theory aimed at summarizing the quali- ties of consciousness that have been discovered since the seminal works of William James (1890). The most important concept is the acknowledgement that consciousness takes place in streams, namely in channels that, computationally speaking, carry a very low bandwidth (information per unit time). Next, we only experience a single stream at a time. Many experiments on sensory rivalry have excluded that we may simultaneously perceive more than one scene, and this fact has been long discussed in terms of biological advantage in speeding up decision for, e.g., gathering food. The conclusion is that consciousness is a serial, low-information process that at any time integrates the massive, unconscious, parallel activity of the majority of brain neurons in a single scene at a time. The GW in this sense is a meta-theory, as its emergence is solid but still not theoretically explained, making it more a metaphor than a model. In a nutshell, there must exist a process, and, maybe a locus for the process, where all information gathers and some of it is selected to reach conscious awareness (Baars, 1988).

The most prominent physiological counterpart for the GW theory is the "reentry theory" of Nobel Laureate G.M. Edelman (1987). Integrated nuclei, i.e. neural circuits kept stable by feedback loops, carry parallel computation, but one central thalamocortical system, with its own re-entrant feedbacks, provides a plausible infrastructure for the $\mathrm{GW}$ (Edelman et al., 2011). It has been recently conjectured that unconsciousness derives from the impairment of feedbacks (Boly et al., 2012). A central role in the reentry theory is played by the complexity of the network infrastructure that supports the GW nucleus (Tononi et al., 1994). We may add that this seminal idea was able to boost the still growing research on human connectome (Sporns, 2014).

A complementary approach, based on information theory, is focused on the dynamical neural-activity patterns occurring over the complex network. Complexity, hence the emerging property of consciousness, is, according to Giulio Tononi, ultimately associated to informational flow (Tononi, 2008; Oizumi et al., 2014). It is the integration of information flow that makes global information different than the sum of its parts. Consciousness is formally defined as this difference, which is zero both when the system is fully synchronous and when its parts do not interact. This theory, called Integrated Information Theory (IIT) successfully explains sleep-related unconsciousness, both during 
NREM sleep where moduli independence has been recently revealed (e.g. by Boly et al 2012), and during propofol-induced anesthesia, when brain activity is strongly synchronized (Lewis et al., 2012). Intuitively, IIT states that a bead of metal, whose electrical activity is dominated by electrons in the conduction band, keeps being unconscious both at rest, with electrons moving due to thermal fluctuations and when synchronously moving, for example when the bead is oscillating as the weight of a simple pendulum.

The pivotal role of the thalamus in sculpting both network and information-flow complexity has been discussed in the review of Ward (2011). Thalamus is composed of a few dozen of nuclei and subnuclei, with negligible neural cross-talk in term of chemical synapses (Hughes et al., 2002; Timofeev and Chauvette, 2011). They indeed project to specific cortical areas and collect information from those. Notice that the thalamic afference is much higher than the corresponding efference: thus, the bandwidth relative to information gathering is much larger than that relative to reactive control. This means that the role of the thalamus is that of integrating incoming information and resending the compressed information back to the different cortical areas, in a feedback loop. Each loop cycle has thus the form of a coarse-grained projection, i.e. the output towards the cortex is coarse-grained (i.e. contains less information) with respect to the input from the cortex to the thalamus. In mathematics, it is well known that any stable solution of an iterative coarse graining process (e.g. a zoom) is a fractal. This discovery, made by Kadanoff (1966), predicts that criticality, which we will introduce in the following paragraph, is associated with scale-free properties (Werner, 2012).

The metaphor of the bead of metal was introduced to intuitively describe the predictive merits of Tononi's IIT. Now we use it to better clarify how mathematical and physical notions of complexity can be transferred into the realm of neurosciences. Imagine that the bead is made of iron or some other ferromagnetic material. This has interesting consequences. First, it is well known that physical memories in electronics exploit ferromagnetism, as the net internal magnetic field depends on the past history of the external one. Above a certain temperature, namely when random fluctuations are too high, all memory is lost. Near this transition temperature, called critical point, we have the emergence of properties closely resembling both the GW and the IIT theory. In other words, as many scientists conjecture, the awake brain resides near a critical point (Chialvo, 2010; Beggs \& Timme, 2012; Massobrio et al., 2015 and references therein). Before entering the discussion, let us define as subcritical a system that behaves as a sum of independent parts, and supercritical a system where all parts behave in a coherent way. As mentioned, IIT would predict low integrated information in both cases. Interestingly, near this transition it would predict high levels of integrated information.

The main properties of a critical system, i.e. a thermodynamic system at the critical point, are the following. 1) As mentioned, information of the whole is different from the sum of the information of the parts. It is impossible, at criticality, to divide the system in two uncorrelated parts.

2) This is in turn due to the dynamical presence of a slowly changing backbone of correlated elements of the system, also called "giant cluster". While the majority of the system does not belong to this backbone, the dimension of the giant cluster spans the whole system in fractal geometry, so that no part of the system is "too far from it". As there is only one giant cluster at a time, we may see it as a serial integrator of parallel activity.

3) Infinite autocorrelation in time and space, i.e. presence of network hierarchical modularity with slowly changing dynamics, namely with a persistency lasting for very extended time durations.

We notice that the conjecture of "critical brain" connects GW and IIT theories. It also connects network complexity to non-extensive entropy production (i.e. redundancy at all scales). Finally, we mention those properties that connect to measurable quantities.

4) Critical systems have infinite susceptibility to external fields, which can be translated into maximal capability of response to external stimuli.

5) The aforementioned persistency, or infinite correlation time in the physical jargon, ultimately stems from fluctuations of macroscopic quantities (in the physical jargon, of the order parameters), so the dynamics of the giant cluster (possibly of the GW) visits metastable states with a diversity of durations, undergoing abrupt transitions from one metastable state to the following. The diversity of these duration times ultimately yields the 1/f properties of the system power spectrum (Allegrini et al., 2009; 2010a).

6) Structural changes are mediated by transitions that propagate with a domino-like evolution. 
However these avalanches have to stay stable, namely not to quench too fast neither to explode in an exponential way (Beggs \& Plenz, 2003).

This latter property, envisaged since the early works of Turing (1950), is normally associated to "selforganized criticality" (SOC), whose paradigm, in the easiest form, is the process of avalanching in a sand pile, kept stable by continuous injection of sand from the top. SOC has been verified, in terms of firing, in practically every neural system, both in vitro and in vivo (for a review, see Massobrio et al., 2015). Our group showed its validity also at the EEG level, in wakefulness and all phases of sleep (Allegrini et al., 2010b, 2013, 2015). Notice, however, that this property, despite much literature on this subject, does not in fact reflect real stable critical conditions, but rather a drive towards auto-organization. This is in fact a short-time property and does not need re-entrant feedbacks to take place. Criticality, on the other hand, refers to a long-time stable or metastable condition, made possible by re-entrant feedbacks.

In Section 4 we conjecture that the global re-entry feedback (pertaining to the giant integrated nucleus playing the role of the $\mathrm{GW}$ ), present in the awake brain, is replaced, during SWS, by the occurring of SSOs.

\section{Sleep Slow Oscillation: from cellular to network level}

At the cellular level, during SWS, cortical and thalamic neurons undergo a dynamical pattern corresponding to an alternation between hyperpolarization, with synaptic silence, and depolarization with synaptic activity and bursting (for a review see Steriade, 2006). The durations of hyperpolarization, also called "down state" and of the bursting segments, also called "up states" are of several hundreds of milliseconds. Up states have been considered as fragments of wakefulness since they show high frequency EEG rhythms and spatiotemporal coherence similar to wakefulness (Sejnowski and Destexhe, 2000; Destexhe et al., 2007). The fast EEG rhythms crowning the up state have been reported as neural correlates of memory consolidation (Molle \& Born, 2011) with mentation as by product (Steriade, 2000). Overall, the process, called neural bistability, lasts more than one second for each neuron (Timofeev et al., 2001) and is due to a shift in the dynamical equilibrium of ionic currents. For instance, in thala- mocortical neurons, the balance between $\mathrm{Ca}^{2+}$ and $\mathrm{K}^{+}$currents becomes multi-stable (more than one membrane-voltage points yielding zero net current) when a leakage current of $\mathrm{K}^{+}$is present. When the $\mathrm{K}^{+}$-current response to voltage differences is high, i.e. with many potassium channels open, there is a single equilibrium point at the $-90 \mathrm{mV}$, the hyperpolarization voltage (Crunelli et al., 2006).

Large neural populations undergo bistability in a coordinated way. This results in a high amplitude electrophysiological response at the level of EEG and MEG. EEG SSO has a peculiar shape: emerging from a zero-centered baseline, it consists of a first positive bump, followed by a huge negative peak followed in turn by a large positive deflection (Massimini et al., 2004; Menicucci et al., 2009; Piarulli et al., 2010). The velocity of descent to the negative peak roughly corresponds to how synchronously neurons fall into the down state (Esser et al., 2007; Menicucci et al., 2009; Carrier et al., 2011). The peak-to-peak amplitude of the SSO, detected on the scalp electrode, is a measure of the numerosity of underlying neural assemblies undergoing bistability (Hanlon et al., 2011). The two measures, taken together, are the EEG correlates of the so-called "proneness to bistability", namely the intensity of the leakage current caused by the opening of activity-dependent potassium channels (Vyazovskiy et al., 2007). As a result, the alternation of up and down states take place at a variety of scales, from the cellular to a large network level.

Our research group has studied the human SSO in depth. In (Menicucci et al., 2009) and in (Piarulli et al., 2010) a detection method to unravel SSOs out of the EEG signal was provided. It is based on the seminal work of Massimini el al. (2004) where the normative algorithm was given: a sharp negative peak of at least $-80 \mathrm{mV}$, and overall amplitude of $140 \mathrm{mV}$. The waves corresponding to these criteria are called full-fledged SSOs. As these waves cooccur with some delay on different EEG electrodes, a single "SSO event" consists of all SSO waves in all channels, namely those displaying full-fledged SSOs complemented with subthreshold ones, whose shape strongly correlates with the full-fledged part of the event. Massimini et al. (2004) showed that SSO is a wave traveling on the cortical mantle in a fronto-posterior direction, with a speed of propagation of the negative-peak average of about $5 \mathrm{~m} / \mathrm{s}$. 
They also showed that the topology of the SSO detection rate and amplitude have a marked frontooccipital gradient, reflecting the homeostatic pressure after daytime wakefulness (Tononi \& Cirelli, 2006).

There has been an intense debate on whether SSO is a pure cortical phenomenon or the result of a thalamocortical interplay. Although the thalamic ablation seems not to fully abolish cortical SSO expression (Timofeev et al., 2000, Lemieux et al., 2014), two seminal reviews (Crunelli \& Hughes, 2010; Crunelli et al., 2015), mainly focused on electrophysiological recordings in animals, stated that the full understanding of SSOs can not be achieved without considering the thalamo-cortical network as a single functional and dynamic unit. Our data on a patient with a selective thalamic degeneration, a consequence of a Fatal Familial Insomnia, indicate a dramatic suppression of SSOs, supporting the hypothesis of a bidirectional cortico-thalamic interplay in generating and modulating the SSO features (Gemignani et al., 2012).

One of the aims of our group has been and still is that of linking thalamic modulation with excitatory activity and the following down states. For this reason, in two recent studies, we focused on episodes of bistability not immediately preceded (by at least 3 seconds) by other SSOs. This choice has allowed the identification of rhythms and network conditions predictive of the falling into the down state. It has been hypothesized that isolated instances of bistability could be triggered by some energy surplus in the form of a wake-like excitatory wave that precedes the down state (Menicucci et al., 2013; Laurino et al., 2014). As the opening of leakage channels is favored, the fact that an excitation is able to induce a transition into the down state is not unexpected both from a theoretical and an experimental point of view. Perturbing a system with two stable states does in general force a transition to the lowest-energy one. This holds true also for the sleeping neurons, as shown in neural models (Wilson et al., 2006; Frohlich et al., 2006). Still at a computational level, depolarization-activated $\mathrm{K}^{+}$channels have been used by Hill \& Tononi (2005) to model bistability in the thalamo-cortical system. Experimentally, this transition to the down state after an excitatory trigger has been described in ferretbrain slices by Sanchez-Vivez et al. (2000, 2010). In synthesis, these potassium channels determine the SSO expression, because:
1) Their opening is blocked by cholinergic activity (Rudolph et al., 2005). This means that SSOs cannot in general take place during wakefulness or REM sleep.

2) Excitatory systems, other than the cholinergic ones, as they increase the network excitability, also increase the homeostatic pressure for sleep, so the more the past activity the more the opening of potassium channel for SSOs (Sanchez-Vivez et al. 2000; Faraguna et al., 2008; Vyazovskiy et al., 2009). This property has been experimentally verified (for a review see Murphy et al., 2011): tasks during wakefulness that activate a particular area correlate with an increase of Slow Wave Activity in the frequency range of SSO in the same area.

3) Local non-cholinergic excitations act as a trigger for the opening of these channels (Amzica and Steriade, 1998a, Eschenko et al., 2012).

At the EEG level, we have shown that a positive bump regularly precedes the negative peak by 300 $500 \mathrm{~ms}$ (Menicucci et al., 2013). Not only does the positive bump significantly emerge upon the zerocrossing line, but it also contains fast EEG rhythms, up to gamma activity. Thus, it indeed acts as a trigger for the $\mathrm{K}+$ channels opening.

In order to better define the temporal dynamics of the relationships between wake-like excitation and the following bistability, we have used the model of sensory evoked responses during NREM sleep, the so-called $\mathrm{K}$ Complexes. We recall that $\mathrm{K}$ Complexes share the same cellular mechanisms taking place in SSOs (Amzica \& Steriade, 1998b, 2002). The K Complex model allowed us to tackle the following questions: Are we unconscious of sensory stimuli during sleep? Are we really insensitive to the external world or some complex process happens? Subjectively, we have all experienced perception while sleeping, e.g. sudden awakenings due to nocturnal earthquakes. Our study clarified how the sleeping brain interacts with external environment. We administered to healthy subjects simple acoustic, tactile and visual stimuli while sleep got deeper. High-density EEG showed that, for 200 milliseconds, sleeping brain starts processing stimuli in specific sensory cortical areas, but this very wake-like activity (P200, or positive bump in spontaneous SSOs) can induce a diffuse half-a-second's electrical silence (N550 or down state) in higher-level cortical areas, which erases consciousness and protects sleep (Laurino et al., 2014). We found that the real travel 
of SSO is guided by that of the excitatory trigger with an opposite direction with respect to the travel of the negative peak, whose travel is in fact apparent and caused by different levels of cortical proneness to bistability in different cortical areas (Laurino et al., 2014). The emerging scenario is that of a bistability driven by the interplay between two necessary conditions: (i) the efficient cortical travel of P200 from primary sensory areas to frontal ones, and (ii) the higher proneness to bistability of these latter areas. As far as proneness to bistability is concerned, we have recently demonstrated that the higher the thalamo-cortical entanglement (expressed by sigma activity), the higher the amplitude and the probability of $\mathrm{SSO}$, both in average and for each event (Menicucci et al., 2015). Indeed the SSO travel occurs on paths previously prepared by higher thalamo-cortical entanglement (Menicucci et al., 2015).

\section{The Sleep Slow Oscillation in the context of theories of consciousness: new vistas towards a synthesis}

We are in a position to conjecture that the role of the down state is to disrupt the interaction between thalamo-cortical entanglement and evoked sensory excitations (or spontaneously integrated excitatory activities) for preventing the emergence of global integration. This, already clear at cellular and network level, fits all aforementioned theories of consciousness with no mutual contradiction. This Section is devoted to clarify the connections of our findings to the aforementioned theories. We will review the stationary or quasi-stationary conditions of the sleeping brain, with strong engagement of the thalamus in preventing firing of cortical neurons and thus interregional information flow. The natural stimulus that encounters this condition is able to modify this pattern, with a complex response at different levels. As sleep onset is outside the scope of this review, we first focus on the sleeping condition before the stimulus. Why are we unconscious? Tononi's IIT has the merit of clarifying that hyper-synchronization is indeed a form of fragmentation, both preventing a consciousness core to emerge. This means that a largely synchronized brain is associated to minimal neural information flow.

During NREM sleep, the thalamus indeed drives large portions of the brain in slowly changing spatiotemporal patterns. This thalamo-cortical entrainment is directly marked by sigma activity (Menicucci et al., 2015). This entrainment may give the false impression that the system is undergoing system integration in the areas involved. In fact, cortico-cortical activity is diminished with respect to wakefulness, at least in terms of firing rate (Timofeev et al., 1996; Vyazovskiy et al. 2009) and thus the information flow is impaired. Pyramidal cells patternization is caused by simultaneous excitation and inhibition from thalamo-cortical projections (DeFelipe \& Fariñas, 1992; for a review, see Sejnowski \& Destexhe, 2000). As a result, their excitability is high, but the firing is inhibited. Thus, communication among neural patches is absent, but their potassium channels are facilitated in their opening (Zhu et al., 2011).

Brain-activity fragmentation has been reported in recent works, both in anesthesia (Lewis et al., 2012) and in natural sleep (Boly et al., 2012; Allegrini et al., 2013). Different nuclei, in particular those pertaining to the Default Mode Network, a network active during inactivity or self-reflection, show higher independence indexes during unconscious states. This lack of inter-nuclei connectivity also explains the results of Massimini et al. (2005) that showed that a transcranial magnetic stimulus (TMS) administered in the premotor cortex only gives rise to a spreading excitation during conscious states, but stay local, with fast quenching in NREM sleep. However, this fragmentation state is presumably a dynamical, rather than a stable state. Different levels of integration, involving different areas, take places during sleep. This is because neural plasticity is unavoidable, even in vitro, with a pressure towards critical integration, as proven by the literature on critical avalanches (Beggs \& Plenz, 2003). We advance the hypothesis that NREM sleep is a transient condition when the brain activity is kept segregated; however the brain tends to a critical state as its thermodynamic equilibrium (Allegrini et al., 2015).

When a natural stimulus is administered, the wellwired thalamo-cortical and cortical architecture regularly allows the creation of large-scale avalanches, with a wake-like wave traveling from primary cortical areas to frontal integrative ones (Laurino et al., 2014). In other words, a natural stimulus and, we conjecture, spontaneous excitations, act as a catalyst to re-instate long-range connectivity. Indeed, this passage elicits cortico-thalamo-cortical re-entrant loops along the excitation travel, via core thalamic projections, 
interfering with the thalamic patternization that was preventing information flow (Sejnowski \& Destexhe, 2000). The emergence of long-range communication drives the brain to a quasi-conscious state. However, this excitation is also responsible for the opening of the potassium channels, with a consequent SSO that, via a half-a-second's neural stillness, resets and disconnects the involved areas.

The above-envisaged picture has the merit of connecting much experimental evidence on SSOs. It is also completely coherent with current theories of consciousness. Before the stimuli, the GW is absent, with low level of integrated information, and with thalamic re-entries that are unable to integrate activity of different areas. The neurons, according to many experimental evidences (Massobrio et al., 2015), still undergo Self-Organized Critical avalanching, but the system is far from a stable critical state. Indeed, we have a pressure towards criticality, and the emergence of an integrated structure is favored (and accompanied) by excitatory activities. These excitations elicit the opening of potassium channels and thus the down states (Sanchez-Vives et al., 2010).

Imagine that the stimulus is strong enough to provoke awakening. This may happen because stimuli also elicit a brain-stem activity that, via the reticular activating system, prevents potassium channels from opening. However, this does not interfere with the integrative dynamics initiated by the stimulus. A high-level connectivity, with re-entrant loops, emerges (Edelman's theory); the thalamus starts its coarsegraining projections (Ward's theory) thus driving the brain towards stable states with high levels of integrated information (Tononi's theory). In other words, a critical Global Workspace (Baars' theory), that serially integrates all brain activity, has formed.

\section{References}

Allegrini P., Menicucci D., Bedini R., Fronzoni L., Gemignani A., Grigolini P., West Bj., Paradisi P. Spontaneous brain activity as a source of ideal 1/f noise. Phys. Rev. E, 80: 061914, 2009.

Allegrini P., Menicucci D., Bedini R., Gemignani A., Paradisi P. Complex intermittency blurred by noise: theory and application to neural dynamics. Phys. Rev. E, 82: 015103, 2010a.

Allegrini P., Paradisi P., Menicucci D., Gemignani A. Fractal complexity in spontaneous EEG meta- stable-state transitions: new vistas on integrated neural dynamics. Front. Physiol. 1: 128, 2010 b.

Allegrini P., Paradisi P., Menicucci D., Laurino M., Bedini R., Piarulli A., Gemignani A. Sleep unconsciousness and breakdown of serial critical intermittency: New vistas on the global workspace. Chaos, Solitons \& Fractals, 55: 32-43, 2013.

Allegrini P., Paradisi P., Menicucci D., Laurino M., Piarulli A., Gemignani A. Self-organized dynamical complexity in human wakefulness and sleep: Different critical brain-activity feedback for conscious and unconscious states. Phys. Rev. E, 92: 032808, 2015.

Amzica F., Steriade M. Electrophysiological correlates of sleep delta waves. Electroencephalogr. Clin. Neurophysiol., 107:69-83, 1998a.

Amzica F., Steriade M. Cellular substrates and laminar profile of sleep K-complex. Neuroscience, 82:671, 1998 b.

Amzica F., Steriade M. The functional significance of K-complexes. Sleep Med. Rev., 6: 139-149, 2002.

Baars B.J. A cognitive theory of consciousness. 1988, Cambridge, UK: Cambridge University Press.

Baars B.J. How does a serial, integrated and very limited stream of consciousness emerge from a nervous system that is mostly unconscious, distributed, parallel and of enormous capacity? Ciba Found Symp., 174: 282-90; discussion 291-303, 1993.

Beggs J.M., Plenz D. Neuronal avalanches in neocortical circuits. J Neurosci., 23: 11167-11177, 2003.

Beggs J.M., Timme N. Being critical of criticality in the brain. Front Physiol., 3:163, 2012.

Boly M., Perlbarg V., Marrelec G., Schabus M., Laureys S., Doyon J., et al. Hierarchical clustering of brain activity during human nonrapid eye movement sleep. Proc. Natl. Acad. Sci., 109: 5856-5861, 2012.

Cahn B.R., Polich J. Meditation states and traits: EEG, ERP, and neuroimaging studies. Psychol. Bull., 132:180-211, 2006.

Carrier J., Viens I., Poirier G., Robillard R., Lafortune M., Vandewalle G., Martin N., Barakat M., Paquet J., Filipini D. Sleep slow wave changes during the middle years of life. Eur. J. Neurosci., 33: 758766, 2011.

Chialvo D.R. Emergent complex neural dynamics. Nat. Phys., 6:744-750, 2010.

Crunelli V., Cope D.W., Hughes S.W. Thalamic T-type Ca2+ channels and NREM sleep. Cell Calcium, 40: 175-190, 2006. 
Crunelli V., Hughes S.W. The slow $(<1 \mathrm{~Hz})$ rhythm of non-REM sleep: a dialogue between three cardinal oscillators. Nat. Neurosci., 13: 9-17, 2010.

Crunelli V., David F., Lőrincz M.L., Hughes S.W. The thalamocortical network as a single slow wave-generating unit. Curr. Opin. Neurobiol., 31: 72-80, 2015.

De Felipe J., Fariñas I. The pyramidal neuron of the cerebral cortex: morphological and chemical characteristics of the synaptic inputs. Prog. Neurobiol., 39: 563-607, 1992.

Destexhe A., Hughes S.W., Rudolph M., Crunelli V. Are corticothalamic 'up' states fragments of wakefulness? Trends Neurosci., 30: 334-342, 2007.

Edelman G.M., Gally J.A., Baars B.J. Biology of consciousness. Front. Psychol., 2: 4, 2011.

Edelman G.M. Neural darwinism: the theory of neuronal group selection. 1987, New York: Basic Books.

Eschenko O., Magri C., Panzeri S., Sara S.J. Noradrenergic neurons of the locus coeruleus are phase locked to cortical up-down states during sleep. Cereb. Cortex, 22: 426-435, 2012.

Esser S.K., Hill S.L., Tononi G. Sleep homeostasis and cortical synchronization: I. Modeling the effects of synaptic strength on sleep slow waves. Sleep, 30: 1617, 2007.

Faraguna U., Vyazovskiy V.V., Nelson A.B., Tononi G., Cirelli C. A causal role for brain-derived neurotrophic factor in the homeostatic regulation of sleep. J. Neurosci., 28: 4088-4095, 2008.

Feinberg T.E. Neuroontology, neurobiological naturalism, and consciousness: a challenge to scientific reduction and a solution. Phys. Life Rev., 9: 13-34, 2012.

Ferri R., Rundo F., Bruni O., Terzano M.G., Stam C.J. The functional connectivity of different EEG bands moves towards small-world network organization during sleep. Clin. Neurophysiol., 119: 2026-2036, 2008.

Fröhlich F., Bazhenov M., Timofeev I., Steriade M., Sejnowski T.J. Slow state transitions of sustained neural oscillations by activity-dependent modulation of intrinsic excitability. J. Neurosci., 26: 6153-6162, 2006.

Gell-Mann M. Consciousness, reduction, and emergence. Some remarks. Ann. N.Y. Acad. Sci., 929: 41-49, 2001.

Gemignani A., Laurino M., Provini F., Piarulli A., Barletta G., d'Ascanio P., Bedini R., Lodi R., Manners D.N., Allegrini P., Menicucci D., Cortelli
P. Thalamic contribution to Sleep Slow Oscillation features in humans: a single case cross sectional EEG study in Fatal Familial Insomnia. Sleep Med., 13: 946-952, 2012.

Kadanoff L.P. Scaling laws for Ising models near $\mathrm{T}_{\mathrm{c}}$, Physics 2: 263, 1966.

Hanlon E.C., Vyazovskiy V.V., Faraguna U., Tononi G., Cirelli C. Synaptic potentiation and sleep need: clues from molecular and electrophysiological studies. Curr. Top Med. Chem., 11: 2472-2482, 2011.

Hughes S.W., Blethyn K.L., Cope D.W., Crunelli V. Properties and origin of spikelets in thalamocortical neurones in vitro. Neuroscience, 110: 395-401, 2002.

James W. The principles of psychology.1890, New York: H. Holt and Company.

Laurino M., Menicucci D., Piarulli A., Mastorci F., Bedini R., Allegrini P., Gemignani A. Disentangling different functional roles of evoked K-complex components: Mapping the sleeping brain while quenching sensory processing. Neuroimage, 86: 433-45, 2014.

Lemieux M., Chen J.Y., Lonjers P., Bazhenov M., Timofeev I. The impact of cortical deafferentation on the neocortical slow oscillation. J. Neurosci., 34: 5689-5703, 2014.

Lewis L.D., Weiner V.S., Mukamel E.A., Donoghue J.A., Eskandar E.N., Madsen J.R., et al. Rapid fragmentation of neuronal networks at the onset of propofol-induced unconsciousness. Proc. Natl. Acad. Sci. U S A, 109: E3377-E3386, 2012.

Massimini M., Huber R., Ferrarelli F., Hill S., Tononi $\mathrm{G}$. The sleep slow oscillation as a traveling wave. J Neurosci., 24: 6862-6870, 2004.

Massimini M., Ferrarelli F., Huber R., Esser S.K., Singh H., Tononi G. Breakdown of cortical effective connectivity during sleep. Science, 309: 22282232, 2005.

Massobrio P., de Arcangelis L., Pasquale V., Jensen H.J., Plenz D. Criticality as a signature of healthy neural systems. Front Syst Neurosci., 9: 22, 2015.

Menicucci D., Piarulli A., Allegrini P., Bedini R., Bergamasco M., Laurino M., Sebastiani L., Gemignani A. Looking for a precursor of spontaneous Sleep Slow Oscillations in human sleep: The role of the sigma activity. Int J Psychophysiol., 97: 99-107, 2015.

Menicucci D., Piarulli A., Allegrini P., Laurino M., Mastorci F., Sebastiani L., Bedini R., Gemignani A. Fragments of wake-like activity frame down- 
states of sleep slow oscillations in humans: new vistas for studying homeostatic processes during sleep. Int J Psychophysiol., 89: 151-157, 2013.

Menicucci D., Piarulli A., Debarnot U., d'Ascanio P., Landi A., Gemignani A. Functional structure of spontaneous sleep slow oscillation activity in humans. PLoS One., 4: e7601, 2009.

Mölle M., Born J. Slow oscillations orchestrating fast oscillations and memory consolidation. Prog Brain Res., 193: 93-110, 2011.

Murphy M., Huber R., Esser S., Riedner B.A., Massimini M., Ferrarelli F., Ghilardi M.F., Tononi G. The cortical topography of local sleep. Curr Top Med Chem., 11: 2438-2446, 2011.

Oizumi M., Albantakis L., Tononi G. From the phenomenology to the mechanisms of consciousness: Integrated Information Theory 3.0. PLoS Comput Biol., 10: e1003588, 2014.

Piarulli A., Menicucci D., Gemignani A., Olcese U., d'Ascanio P., Pingitore A., Bedini R., Landi A. Likeness-based detection of sleep slow oscillations in normal and altered sleep conditions: application on low-density EEG recordings. IEEE Trans Biomed Eng., 57: 363-372, 2010.

Rudolph M., Pelletier J.G., Paré D., Destexhe A. Characterization of synaptic conductances and integrative properties during electrically induced EEG-activated states in neocortical neurons in vivo. J Neurophysiol., 94: 2805-2821, 2005.

Sanchez-Vives M.V., McCormick D.A. Cellular and network mechanisms of rhythmic recurrent activity in neocortex. Nat Neurosci., 3: 1027-1034, 2000.

Sanchez-Vivez M.V., Mattia M., Compte A., PerezZabalza M., Winograd M., Descalzo V.F., Reig R. Inhibitory modulation of cortical up states. $J$. Neurophysiol., 104: 1314.1324, 2010.

Sejnowski T.J., Destexhe A. Why do we sleep? Brain Res., 886: 208-223, 2000.

Sporns O. Contributions and challenges for network models in cognitive neuroscience. Nat. Neurosci., 17: 652-660, 2014.

Steriade M. Corticothalamic resonance, states of vigilance and mentation. Neuroscience, 101: 243276, 2000.

Steriade M. Grouping of brain rhythms in corticothalamic systems. Neuroscience, 137: 1087-1106, 2006.

Timofeev I., Chauvette S. Thalamocortical oscillations: local control of EEG slow waves. Curr. Top Med. Chem., 11: 2457-2471, 2011.
Timofeev I., Contreras D., Steriade M. Synaptic responsiveness of cortical and thalamic neurons during various phases of slow sleep oscillation in cat. J. Physiol. 494: 265-278, 1996.

Timofeev I., Grenier F., Bazhenov M., Sejnowski T.J., Steriade M. Origin of slow cortical oscillations in deafferented cortical slabs. Cereb. Cortex, 10: 1185-1199, 2000.

Timofeev I., Grenier F., Steriade M. Disfacilitation and active inhibition in the neocortex during the natural sleep-wake cycle. Proc. Natl. Acad. Sci. U $S$ A, 98: 1924-1929, 2001.

Tononi G., Koch C. The neural correlates of consciousness: an update. Ann. N.Y. Acad. Sci., 1124: 239-261, 2008.

Tononi G., Sporns O., Edelman G.M. A measure for brain complexity: relating functional segregation and integration in the nervous system. Proc Natl Acad Sci U S A, 91: 5033-5037, 1994.

Tononi G. Consciousness as integrated information: a provisional manifesto. Biol. Bull., 215: 216-242, 2008

Tononi G., Cirelli C. Sleep function and synaptic homeostasis. Sleep Med. Rev., 10: 49-62, 2006.

Turing A.M. Computing machinery and intellingence. Mind, 59: 433-460, 1950.

Vyazovskiy V.V., Olcese U., Lazimy Y.M., Faraguna U., Esser S.K., Williams J.C., Cirelli C., Tononi G. Cortical firing and sleep homeostasis. Neuron, 63 : 865-878, 2009.

Vyazovskiy V.V., Riedner B.A., Cirelli C., Tononi G. Sleep homeostasis and cortical synchronization: II. A local "eld potential study of sleep slow waves in the rat. Sleep, 30:1631, 2007.

Ward L.M. The thalamic dynamic core theory of conscious experience. Conscious. Cogn., 20: 46486, 2011.

Werner G. From brain states to mental phenomena via phase space transitions and renormalization group transformation: proposal of a theory. Cogn. Neurodyn., 6: 199-202, 2012.

Wilson M.T., Steyn-Ross D.A., Sleigh J.W., SteynRoss M.L., Wilcocks L.C., Gillies IP. The K-complex and slow oscillation in terms of a mean-field cortical model. J. Comput. Neurosci. 21: 243-257, 2006.

Zhu J., Jiang M., Yang M., Hou H., Shu Y. Membrane potential-dependent modulation of recurrent inhibition in rat neocortex. PLoS Biol. 9: e1001032, 2011. 